\title{
Neuropathic pain in knee osteoarthritis
}

\author{
Uğur Güngör Demir ${ }^{1 *} \mathbb{D}$, Ali Nail Demir ${ }^{2}$ and Naciye Füsun Toraman ${ }^{3}$
}

\begin{abstract}
Background: This study aimed to investigate the relationship between the neuropathic pain in knee osteoarthritis with the body composition, anthropometric and postural features, physical function, and quality of life.

Methods: Patients with primary knee osteoarthritis, 50-70 years of age, were included in the study and divided into Group 1 with neuropathic pain and Group 2 with no neuropathic pain according to Douleur Neuropathique-4. The groups were compared in terms of demographic, clinical, radiological, laboratory findings and anthropometric measurements, body composition, physical function tests, osteoarthritis severity, quality of life, and posturography.

Results: 200 patients were included in the study. 98 (82.6\% female) were in Group 1 and 102 (74.5\% female) in Group 2. Age was higher in Group 1 compared to Group 2 [61 (50-70) and 57.5 (50-70), respectively, $p=0.03]$. Symptom duration was also longer in Group 1 (5.21 \pm 4.76 and $3.38 \pm 3.58, p=0.002)$. Body mass indices were $31.9 \pm 5.6$ and $30.1 \pm 4.8(p=0.015)$. Kellgren-Lawrence class, Western Ontario and McMaster Osteoarthritis Index and Short Form36 scores were more unfavorable in Group 1. Although fall risk was similar, stability and Fourier harmony indices were impaired in Group 1 compared to Group 2 especially when the visual and proprioceptive input was blocked.
\end{abstract}

Conclusions: Almost half of the patients with knee osteoarthritis had neuropathic pain which was associated with longer symptom duration and higher age, lower education, higher body mass index, more severe radilogical findings, worse pain perception, lower physical function and quality of life, and lower stability.

Keywords: Knee osteoarthritis, Neuropathic pain, Posturography

\section{Introduction}

Osteoarthritis, which most commonly affects the knee, is a degenerative disease characterized by cartilage erosion, bony hypertrophy, subchondral sclerosis, and synovial and capsular changes [1]. Symptomatic knee osteoarthritis is common in the elderly with a prevalence of $13 \%$ in females and $10 \%$ in males over the age 60 [2, $3]$. Pain is usually the presenting and the most common symptom and speculated to have nociceptive and neuropathic components along with sensitization that can be both mechanical (peripheral) and central (secondary hyperalgesia) [4, 7]. The prevalence of neuropathic pain

\footnotetext{
*Correspondence: ugurgungor90@gmail.com

${ }^{1}$ Department of Physical Therapy and Rehabilitation, University of Health

Sciences, Antalya Training and Research Hospital, 07100 Muratpaşa,

Antalya, Turkey

Full list of author information is available at the end of the article
}

was reported to be $5.4-52 \%$ in knee osteoarthritis [8, $12]$. Neuropathic pain was reported to be associated with age, sex, body mass index, education and working status, radiological osteoarthritis severity, symptom duration, and presence of comorbid diseases in patients with knee osteoarthritis [8-13]. It also has impact on quality of life $[12,13]$. Although the body composition, anthropometric measurements, postural stability, and physical function may have a potential relationship with the neuropathic component of the pain in knee osteoarthritis, they were not reported to be assessed together in that regard.

This study primarily aimed to investigate the relationship between the neuropathic pain in knee osteoarthritis with the body composition, anthropometric and postural features, physical function, and quality of life.

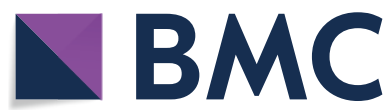

(c) The Author(s) 2021. Open Access This article is licensed under a Creative Commons Attribution 4.0 International License, which permits use, sharing, adaptation, distribution and reproduction in any medium or format, as long as you give appropriate credit to the original author(s) and the source, provide a link to the Creative Commons licence, and indicate if changes were made. The images or other third party material in this article are included in the article's Creative Commons licence, unless indicated otherwise in a credit line to the material. If material is not included in the article's Creative Commons licence and your intended use is not permitted by statutory regulation or exceeds the permitted use, you will need to obtain permission directly from the copyright holder. To view a copy of this licence, visit http://creativecommons.org/licenses/by/4.0/. 


\section{Methods}

\section{Patients and design}

All patients admitted to the Physical Therapy and Rehabilitation Outpatient Clinic, Antalya Training and Research Hospital, between January 2018-January 2019, with the complaint of knee pain, were screened for the eligibility for the study. The inclusion criteria were (a) being between 50 and 70 years of age (b) knee pain in more than 25 days of during the last month (c) crepitation during movement (d) morning stiffness less than $30 \mathrm{~min}$ or radiographic osteophytes (e) Kellgren-Lawrence class I-IV knee osteoarthritis and (f) willingness to participate in the study. The exclusion criteria were (a) previous knee operation (b) rheumatic or metabolic disease (c) malignant disease (d) central or peripheral neurologic disease that may cause neuropathic pain (e) cardiopulmonary disease that may interfere with the functional tests (f) pregnancy (g) active infections (h) a standardized mini-mental status examination score of less than 26 (i) intra-articular steroid injection in the last 3 months (j) hip or ankle pathology (k) treatment for neuropathic pain in the last 6 months.

Age, sex, education, working, smoking, and marital status, symptom duration, number of comorbid diseases and drugs used, and painful areas other than knee were recorded for each patient along with the presence of postural deviations (thoracic hyperkyphosis, lombar hyperlordosis, scoliosis, genu varum and recurvatum, and pes planus).

\section{Tests and measurements: anthropometric measurements and body composition}

Standing height was measured using a wall-mounted stature meter. Waist and hip circumferences were measured using a tape measure. Weight, body mass index and composition were measured using a professional digital scale (Tanita ${ }^{\circledR} \mathrm{MC}-180 \mathrm{MA}$, Tanita Corp., Tokyo, Japan).

\section{Western Ontario and McMaster Osteoarthritis Index (WOMAC)}

It is a health status instrument with pain, stiffness and physical function domains designed for patients with osteoarthritis and validated in Turkish population [14, $15]$.

\section{Short form-36 (SF-36)}

It is a 36-item questionnaire for general health and quality of life and divided into eight domains: physical function, social function, physical role limitations, emotional role limitations, bodily pain, general mental health, vitality, and general health perceptions [16, 17].

\section{Geriatric depression scale}

It is a 30-item scale first created by Yesavage et al. [18] and validated in Turkish population [19]. A score of 0-10 was considered as no depression, $11-13$ as possible depression, and 14 or higher as depression.

\section{Physical function}

A 30-s chair-stand, stair-climbing, and 6-min walk tests were performed for each patient as described previously [20-22].

\section{Posturographic evaluation}

Computerized tetra-ataxiometric posturography, Tetrax ${ }^{\circledR}$ (Sunlight Medical Ltd., Tel Aviv, Israel), was used for the assessment of static posturographic stability in eight positions: stable surface/eyes open/face forward, stable surface/eyes closed/face forward, unstable surface/ eyes open/face forward, unstable surface/eyes closed/ face forward, stable surface/eyes closed/head rotated $45^{\circ}$ to the right, stable surface/eyes closed/head rotated $45^{\circ}$ to the left, stable surface/eyes closed/head tilted back $30^{\circ}$, and stable surface/eyes closed/head tilted $30^{\circ}$ forward. Fall risk index (low: 0-36, intermediate: 37-58, high: 59 or higher), stability index (the higher the index the lower the stability), and Fourier harmony index (<0.9: abnormal stability; normal: 0.9-1) were calculated for each patient $[23,24]$.

\section{Douleur neuropathique-4 (DN-4)}

It is a scale composed of four questions. A score of 4 or higher indicates neuropathic pain $[25,26]$. Additionally, a ten centimeter visual analogue scale (VAS) was used for the severity of the knee pain at rest and while walking $(0=$ no pain, $10=$ extreme pain $)$.

\section{Radiological assessment}

A standard standing anteroposterior knee X-ray with the knees in extension were obtained for each patient and severity of osteoarthritis was graded according to the Kellgren-Lawrence classification [27].

\section{Laboratory analysis}

Hemoglobin concentration, erythrocyte sedimentation rate and serum $\mathrm{C}$-reactive protein, fasting plasma glucose and lipid profile (total cholesterol, LDL cholesterol, and triglyceride), serum uric acid, creatinine, and alanine aminotransferase, plasma 25-hydroxy-vitamin- $\mathrm{D}_{3}$ and thyroid stimulating hormone levels of each patient were measured. 


\section{Study design}

The patients were divided into two groups according to the DN-4 score: Group 1 with neuropathic pain (a DN-4 score of 4 or higher) and Group 2 with no significant neuropatic pain (a DN-4 score less than 4 ). The two groups were compared in terms of the demographic and clinical features and the tests and measurements. Since the sex was found to have a influencer effect, the study groups were further divided into the sex subgroups in the analyses.

\section{Statistical analysis}

SPSS Statistics v.18 (IBM Corp, Chicago, IL, USA) was used for the statistical analyses. Data were expressed as numbers with percentages for the categorical and means or medians with standard deviations or minimum and maximum values for the continuous variables. Categorical variables were compared by using chi-square of Fisher's exact tests. Distributions of the continuous data were analyzed by histograms and tested for normality by the Shapiro-Wilk test. Mann-Whitney U or a t-test was used for comparison of unrelated samples according to the distribution. Multiple logistic regression analysis was used to control for the factors associated with neuropathic pain. $p<0.05$ was considered statistically significant.

\section{Results}

A total of 200 patients were included in the study (Fig. 1). Demographic, clinical, and radiological features of the study groups according to sex were given in Table 1 . There were 81 (82.6\%) and 76 (74.5\%) females in Group

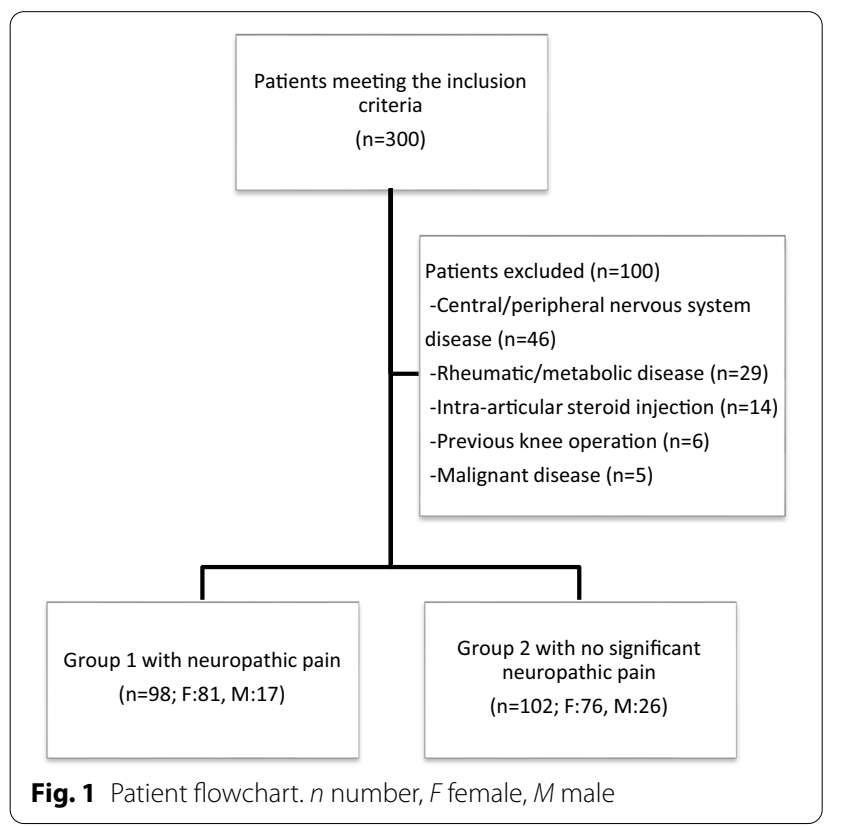

1 and Group 2, respectively ( $p=0.161)$. Age was slightly higher in Group 1 and education status was worse in that group. Symptom duration was significantly longer in Group 1 compared to that of Group 2 (5.21 \pm 4.76 vs. $3.38 \pm 3.58$ years, respectively). Kellgren-Lawrence classes were more unfavorable and genu varum was more frequent in Group 1 (Table 1). The mean DN-4 scores, according to which the patients were grouped, were $6.21 \pm 1.47$ and $1.16 \pm 1.26$ in Group 1 and Group 2 , respectively. In both groups females had higher DN-4 scores compared to those of males but the differences did not reach to a level of statistical significance $(6.35 \pm 1.46$ vs. $5.59 \pm 1.42, p=0.053$ and $1.25 \pm 1.3$ vs. $0.88 \pm 1.11$, $p=0.171$ in Group 1 and Group 2, respectively). Anthropometric measurements and body composition of the study groups were given in Table 2 . Body mass index and body fat percentage were also slightly higher in Group 1 compared to those of Group 2. As shown in Table 3, physical function, general health status, pain perception, and quality of life indices were all worse in Group 1. Fall risk, stability and Fourier harmony indices obtained by the computerized posturography in eight positions were provided in Table 4. Fall risk was found to be higher in males in Group 1 compared to that of males in Group 2, although there was no difference between females. Stability indices in different positions were also more unfavorable in males in Group 1 (Table 4). The rates of abnormal Fourier harmony index (i.e. less than 0.9) in eight positions were similarly distributed between the study groups (47-69\% in Group 1 and 52-65\% in Group 2) and no sex difference was present (data not shown; see Table 4 for the absolute index values). Among age, education status, symptom duration, Kellgren-Lawrence class, number of comorbid diseases and drugs, body mass index, and depression scale score, which were found to be associated with neuropathic pain (Tables 1, 2 and 3), symptom duration $(p=0.01)$, depression scale score $(p<0.001)$, and Kellgren-Lawrence class $(p=0.02)$ remained significant to predict neuropathic pain in multiple logistic regression.

Hemoglobin concentration, erythrocyte sedimentation rate and serum $\mathrm{C}$-reactive protein, fasting plasma glucose and lipid profile, serum uric acid, alanine aminotransferase, and plasma thyroid stimulating hormone levels were similar in Group 1 and Group 2 (data not shown). Plasma 25-hydroxy-vitamin- $\mathrm{D}_{3}$ was slightly lower in Group 1 compared to Group 2 (median 19.6 [min:5, $\max : 76.3$ ] and 22.3 [min:4, max:66] $\mathrm{ng} / \mathrm{mL}$, respectively, $p=0.035$ ). Serum creatinine was also slightly lower in Group 1 compared to Group 2 (median 0.77 [min:0.48, $\max : 1.41]$ and 0.81 [min:0.54, $\max : 1.49] \mathrm{mg} / \mathrm{dL}$, respectively, $p=0.034$ ). But in the sex-stratified comparisons plasma 25-hydroxy-vitamin- $\mathrm{D}_{3}$ and serum creatinine 
Table 1 Demographic, clinical, and radiological features of the study groups

\begin{tabular}{|c|c|c|c|c|c|c|c|c|c|}
\hline & \multicolumn{3}{|l|}{ Group 1} & \multicolumn{3}{|l|}{ Group 2} & \multirow[t]{2}{*}{$p_{F}$} & \multirow[t]{2}{*}{$\mathrm{p}_{\mathrm{M}}$} & \multirow[t]{2}{*}{$\mathrm{p}_{\mathrm{G}}$} \\
\hline & Female $(n=81)$ & Male $(n=17)$ & Total $(\mathrm{n}=98)$ & Female $(n=76)$ & Male $(n=26)$ & Total $(n=102)$ & & & \\
\hline Age, years* & $61(50-70)$ & $60.5(51-70)$ & $61(50-70)$ & $56.5(50-70)$ & $59(50-69)$ & $57.5(50-70)$ & 0.010 & 0.842 & 0.030 \\
\hline $\begin{array}{l}\text { Education status, } \\
\mathrm{n}(\%)\end{array}$ & $\mathrm{N} / \mathrm{P}$ & $\mathrm{N} / \mathrm{P}$ & & $\mathrm{N} / \mathrm{P}$ & $\mathrm{N} / \mathrm{P}$ & & N/P & N/P & 0.014 \\
\hline Primary or lower & & & $56(57.1)$ & & & $44(43.1)$ & & & \\
\hline $\begin{array}{l}\text { Secondary or high } \\
\text { school }\end{array}$ & & & $32(32.7)$ & & & $32(31.4)$ & & & \\
\hline Higher education & & & $10(10.2)$ & & & $26(25.5)$ & & & \\
\hline Working status, n (\%) & $\mathrm{N} / \mathrm{P}$ & $\mathrm{N} / \mathrm{P}$ & & $\mathrm{N} / \mathrm{P}$ & $\mathrm{N} / \mathrm{P}$ & & N/P & $N / P$ & 0.555 \\
\hline Actively working & & & $15(15.3)$ & & & $14(13.7)$ & & & \\
\hline Not working & & & $83(84.7)$ & & & $88(86.3)$ & & & \\
\hline Marital status, n (\%) & $N / P$ & $N / P$ & & $N / P$ & $\mathrm{~N} / \mathrm{P}$ & & N/P & $N / P$ & 0.873 \\
\hline Married & & & 77 (78.6) & & & $89(87.3)$ & & & \\
\hline Not married & & & $21(21.4)$ & & & $13(12.7)$ & & & \\
\hline $\begin{array}{l}\text { Smoking status, } \\
\text { n (\%) }\end{array}$ & $N / P$ & $N / P$ & & $\mathrm{~N} / \mathrm{P}$ & $N / P$ & & N/P & $N / P$ & 0.626 \\
\hline Active smoker & & & $12(12.2)$ & & & $13(12.7)$ & & & \\
\hline Ex-smoker & & & $32(32.7)$ & & & $27(26.5)$ & & & \\
\hline Never smoked & & & $54(55.1)$ & & & $62(60.8)$ & & & \\
\hline Knee pain, n (\%) & & & & & & & 0.308 & 0.337 & 0.904 \\
\hline Unilateral & $13(16.1)$ & $3(17.6)$ & $16(16.3)$ & $8(10.5)$ & $8(30.8)$ & $16(15.7)$ & & & \\
\hline Bilateral & $68(83.9)$ & $14(82.4)$ & $82(83.7)$ & $68(89.5)$ & $18(69.2)$ & $86(84.3)$ & & & \\
\hline $\begin{array}{l}\text { Knee crepitation, } \\
\text { n (\%) }\end{array}$ & & & & & & & 0.234 & 0.136 & 0.059 \\
\hline Unilateral & $6(7.4)$ & $1(5.9)$ & $7(7.1)$ & $10(13.2)$ & $6(23.1)$ & $16(15.7)$ & & & \\
\hline Bilateral & 75 (92.6) & $16(94.1)$ & $91(92.9)$ & $66(86.8)$ & $20(76.9)$ & $86(84.3)$ & & & \\
\hline $\begin{array}{l}\text { Symptom duration, } \\
\text { years }\end{array}$ & $5.54 \pm 4.99$ & $3.65 \pm 3.15$ & $5.21 \pm 4.76$ & $3.8 \pm 3.79$ & $2.14 \pm 2.54$ & $3.38 \pm 3.58$ & 0.015 & 0.108 & 0.002 \\
\hline \multicolumn{10}{|l|}{$\begin{array}{l}\text { Kellgren-Lawrence } \\
\text { classification, } \mathrm{n}(\%)\end{array}$} \\
\hline Right & & & & & & & 0.133 & 0.170 & 0.016 \\
\hline Class I & $7(8.6)$ & $1(5.9)$ & $8(8.2)$ & $13(17.1)$ & $9(34.6)$ & $22(21.6)$ & & & \\
\hline Class II & $26(32.1)$ & $9(52.9)$ & $35(35.7)$ & $29(38.2)$ & $11(42.3)$ & $40(39.2)$ & & & \\
\hline Class III & $34(42)$ & $6(35.3)$ & $40(40.8)$ & $28(36.8)$ & $5(19.2)$ & $33(32.4)$ & & & \\
\hline Class IV & $14(17.3)$ & $1(5.9)$ & $15(15.3)$ & $6(7.9)$ & $1(3.9)$ & $7(6.8)$ & & & \\
\hline Left & & & & & & & 0.001 & 0.286 & 0.001 \\
\hline Class I & $1(1.2)$ & $2(11.8)$ & $3(3.1)$ & 15 (19.7) & $6(23.1)$ & $21(20.6)$ & & & \\
\hline Class II & $28(34.7)$ & $10(58.8)$ & $38(38.7)$ & 27 (35.6) & $15(57.7)$ & $42(41.2)$ & & & \\
\hline Class III & $34(41.9)$ & $5(29.4)$ & $39(39.8)$ & $25(32.9)$ & $3(11.5)$ & $28(27.4)$ & & & \\
\hline Class IV & $18(22.2)$ & - & $18(18.4)$ & $9(11.6)$ & $2(7.7)$ & $11(10.8)$ & & & \\
\hline $\begin{array}{l}\text { Painful areas other } \\
\text { than knee, } n(\%)\end{array}$ & & & & & & & $<0.001$ & 0.028 & $<0.001$ \\
\hline Absent & $11(13.6)$ & $6(35.2)$ & $17(17.3)$ & $36(47.4)$ & $18(69.2)$ & $54(52.9)$ & & & \\
\hline Present & 70 (86.4) & $11(64.8)$ & $81(82.7)$ & $40(52.6)$ & $8(30.8)$ & $48(47.1)$ & & & \\
\hline \multicolumn{10}{|l|}{$\begin{array}{l}\text { Postural deviations, } \\
\text { n (\%) }\end{array}$} \\
\hline $\begin{array}{l}\text { Thoracic hyperky- } \\
\text { phosis }\end{array}$ & $28(34.7)$ & $8(47.1)$ & $36(36.7)$ & 25 (32.9) & $8(30.8)$ & $33(32.3)$ & 0.825 & 0.280 & 0.515 \\
\hline $\begin{array}{l}\text { Lombar hyperlor- } \\
\text { dosis }\end{array}$ & $44(54.3)$ & $10(58.8)$ & $54(55.1)$ & $39(51.3)$ & $13(50)$ & $52(51)$ & 0.706 & 0.571 & 0.559 \\
\hline Scoliosis & $8(9.8)$ & $1(5.9)$ & $9(9.2)$ & $8(10.5)$ & - & $8(7.8)$ & 0.893 & $\mathrm{~N} / \mathrm{T}$ & 0.734 \\
\hline Genu varum & 24 (29.6) & $3(17.6)$ & $27(27.5)$ & $10(13.2)$ & $3(11.5)$ & $13(12.7)$ & 0.012 & 0.666 & 0.009 \\
\hline
\end{tabular}


Table 1 (continued)

\begin{tabular}{|c|c|c|c|c|c|c|c|c|c|}
\hline & \multicolumn{3}{|l|}{ Group 1} & \multicolumn{3}{|l|}{ Group 2} & \multirow[t]{2}{*}{$p_{F}$} & \multirow[t]{2}{*}{$\mathrm{p}_{\mathrm{M}}$} & \multirow[t]{2}{*}{$p_{G}$} \\
\hline & Female $(n=81)$ & Male $(n=17)$ & Total $(n=98)$ & Female $(n=76)$ & Male $(n=26)$ & Total $(n=102)$ & & & \\
\hline Genu recurvatum & - & - & - & - & $1(3.9)$ & $1(1)$ & $N / T$ & $N / T$ & $\mathrm{~N} / \mathrm{T}$ \\
\hline Pes planus & $9(11.1)$ & $2(11.8)$ & $11(11.2)$ & $8(10.5)$ & $3(11.5)$ & $11(10.8)$ & 0.906 & 1.000 & 0.921 \\
\hline $\begin{array}{l}\text { Number of comor- } \\
\text { bid diseases* }\end{array}$ & $3(0-8)$ & $2(0-4)$ & $3(0-8)$ & $2(0-6)$ & $1(0-6)$ & $2(0-6)$ & $<0.001$ & 0.083 & $<0.001$ \\
\hline $\begin{array}{l}\text { Number of drugs } \\
\text { used* }\end{array}$ & $2(0-5)$ & $2(0-3)$ & $2(0-5)$ & $1(0-4)$ & $1(0-3)$ & $1(0-4)$ & $<0.001$ & 0.234 & $<0.001$ \\
\hline
\end{tabular}

$P$-value less than $0.05(p<0.05)$ is statistically significant

$\mathrm{p}_{\mathrm{F}}$; significance of difference between females in Group 1 and Group 2. $\mathrm{p}_{\mathrm{M}}$; significance of difference between males in Group 1 and Group 2. $\mathrm{p}_{\mathrm{G}}$; significance of difference between Group 1 and Group 2

$n$ number, $N / T$ not tested, $N / P$ not provided

* Given as median (min-max)

Table 2 Anthropometric measurements and body composition of the study groups

\begin{tabular}{|c|c|c|c|c|c|c|c|c|c|}
\hline & \multicolumn{3}{|l|}{ Group 1} & \multicolumn{3}{|l|}{ Group 2} & \multirow[t]{2}{*}{$\mathrm{p}_{\mathrm{F}}$} & \multirow[t]{2}{*}{$\mathrm{p}_{\mathrm{M}}$} & \multirow[t]{2}{*}{$\mathrm{p}_{\mathrm{G}}$} \\
\hline & Female $(n=81)$ & Male $(n=17)$ & Total $(n=98)$ & Female $(n=76)$ & Male $(n=26)$ & Total $(n=102)$ & & & \\
\hline Weight, kg* & $74.9(52.4-118.8)$ & $81.4(59-102.4)$ & $76.2(52.4-118.8)$ & $75.9(53.5-110.8)$ & $80.2(59.6-117.4)$ & $76.4(53.5-117.4)$ & 0.572 & 0.297 & 0.488 \\
\hline Height, cm* & $155(141-178)$ & $170(160-182)$ & $157(141-182)$ & $157(140-175)$ & $167(155-179)$ & $159(140-179)$ & 0.016 & 0.432 & 0.026 \\
\hline $\begin{array}{l}\text { Body mass } \\
\text { index, } \mathrm{kg} / \mathrm{m}^{2}\end{array}$ & $32.6 \pm 5.7$ & $28.8 \pm 3.8$ & $31.9 \pm 5.6$ & $30.8 \pm 5$ & $28.2 \pm 3.8$ & $30.1 \pm 4.8$ & 0.037 & 0.613 & 0.015 \\
\hline $\begin{array}{l}\text { Waist circumfer- } \\
\text { ence, } c m\end{array}$ & $102.8 \pm 11.7$ & $103.5 \pm 10.3$ & $102.9 \pm 11.4$ & $99.3 \pm 10.8$ & $102.6 \pm 8.4$ & $100.1 \pm 10.3$ & 0.054 & 0.767 & 0.073 \\
\hline $\begin{array}{l}\text { Hip circumfer- } \\
\text { ence, } \text { cm* }^{*}\end{array}$ & $114(92-144)$ & $107(92-115)$ & $112(92-144)$ & $113(94-142)$ & $104(92-120)$ & $109(92-142)$ & 0.137 & 0.419 & 0.055 \\
\hline $\begin{array}{l}\text { Waist-to-hip } \\
\text { ratio }\end{array}$ & $0.88 \pm 0.07$ & $0.97 \pm 0.06$ & $0.9 \pm 0.07$ & $0.88 \pm 0.05$ & $0.97 \pm 0.04$ & $0.9 \pm 0.06$ & 0.672 & 0.958 & 0.691 \\
\hline $\begin{array}{l}\text { Muscle mass, } \\
\mathrm{kg}^{*}\end{array}$ & $45.6(35.6-64.9)$ & $60.9(51.8-73.9)$ & $46.8(35.6-73.9)$ & $46(33.3-64)$ & $58.8(47.5-78.5)$ & $48.3(33.3-78.5)$ & 0.684 & 0.196 & 0.400 \\
\hline Fat mass, kg* & $26.3(13.6-58.1)$ & $20.6(3.4-30.6)$ & $25.3(3.4-58.1)$ & $27.3(7.8-49.8)$ & 15.7 (6.9-34.9) & $24.6(6.9-49.8)$ & 0.274 & 0.358 & 0.055 \\
\hline Body fat, \%* & $36.7(24.5-48.9)$ & $23.8(5.7-29.9)$ & $34(5.7-48.9)$ & $36.2(10.4-45)$ & $21.7(11.6-29.7)$ & $33.3(10.4-45)$ & 0.236 & 0.559 & 0.039 \\
\hline
\end{tabular}

$P$-value less than $0.05(p<0.05)$ is statistically significant

$\mathrm{p}_{\mathrm{F}}$; significance of difference between females in Group 1 and Group 2. $\mathrm{p}_{\mathrm{M}}$; significance of difference between males in Group 1 and Group 2. $\mathrm{p}_{\mathrm{G}}$; significance of difference between Group 1 and Group 2

$n$ number

* Given as median (min-max)

were similarly distributed between the groups (data not shown).

\section{Discussion}

We were able to show that almost half of the patients with knee osteoarthritis had neuropathic pain which was associated with longer symptom duration and higher age, lower education, higher body mass index, higher number of comorbid diseases and drugs used, presence of genu varum, and more severe radilogical findings. Neuropathic pain was also found to be related to the presence of painful areas other than the knee, worse pain perception, depression, lower physical function and quality of life, and lower stability when the visual and proprioceptive input was blocked.

Osteoarthritis is the most common joint disease and an important cause of mobidity [2]. The principal symptom in osteoarthritis is pain which was proposed to have nociceptive and neuropathic components [6]. Early peripheral (mechanical) and late central sensitization (secondary hyperalgesia) may augment pain perception [5-7]. The latter may predominate in some patients and the pain can not be explained entirely by nociceptive and 
Table 3 Physical function tests, Western Ontario and McMaster Osteoarthritis Index (WOMAC), Short Form-36 (SF-36), geriatric depression scale (GDS), and knee pain visual analogue scale (VAS) scores of the study groups

\begin{tabular}{|c|c|c|c|c|c|c|c|c|c|}
\hline & \multicolumn{3}{|l|}{ Group 1} & \multicolumn{3}{|l|}{ Group 2} & \multirow[t]{2}{*}{$\mathrm{p}_{\mathrm{F}}$} & \multirow[t]{2}{*}{$\mathrm{p}_{\mathrm{M}}$} & \multirow[t]{2}{*}{$p_{G}$} \\
\hline & Female $(n=81)$ & Male $(n=17)$ & Total $(n=98)$ & Female $(n=76)$ & Male $(n=26)$ & Total $(n=102)$ & & & \\
\hline \multicolumn{10}{|l|}{$\begin{array}{l}\text { Physical function } \\
\text { tests* }\end{array}$} \\
\hline Chair-stand /30 s & $11(2-16)$ & $12(6-14)$ & $11(2-16)$ & $12(5-22)$ & $14(8-19)$ & $12(5-22)$ & 0.002 & 0.001 & $<0.001$ \\
\hline \multicolumn{10}{|l|}{$\begin{array}{l}\text { Stair climbing, } \\
\text { second }\end{array}$} \\
\hline Upwards & $2.8(1.6-21.7)$ & $2.4(1.4-4.7)$ & $2.7(1.4-21.7)$ & $2.3(1.2-9.4)$ & $2(1.4-3.8)$ & $2.2(1.2-9.4)$ & 0.003 & 0.160 & $<0.001$ \\
\hline Downwards & $3(1.5-15.2)$ & $2.5(1.4-5.1)$ & $3(1.4-12.5)$ & $2.3(1.3-8.5)$ & $2(1.4-4.3)$ & $2.3(1.3-8.5)$ & $<0.001$ & 0.168 & $<0.001$ \\
\hline $\begin{array}{l}\text { 6-min walk, } \\
\text { meter }\end{array}$ & $360(70-510)$ & $425(240-600)$ & $360(70-600)$ & $420(108-600)$ & $420(280-620)$ & $430(108-620)$ & $<0.001$ & 0.149 & $<0.001$ \\
\hline \multicolumn{10}{|l|}{ WOMAC score* } \\
\hline Pain & $10(3-19)$ & $8(2-16)$ & $10(2-19)$ & $7(1-15)$ & $4(2-12)$ & $6(1-15)$ & $<0.001$ & 0.008 & 0.002 \\
\hline Stiffness & $2(0-7)$ & $0(0-6)$ & $2(0-7)$ & $0(0-4)$ & $0(0-4)$ & $0(0-4)$ & $<0.001$ & 0.275 & $<0.001$ \\
\hline Physical function & $32(12-59)$ & $27(7-55)$ & $30(7-59)$ & $22.5(1-46)$ & $17(5-38)$ & $22(1-46)$ & $<0.001$ & 0.012 & $<0.001$ \\
\hline Total & $12.3(3.9-26.9)$ & $9.2(2-23.5)$ & $11.5(2-26.9)$ & $7.4(1.2-17.2)$ & $5.5(2.1-15)$ & $6.8(1.2-17.2)$ & $<0.001$ & 0.012 & $<0.001$ \\
\hline \multicolumn{10}{|l|}{ SF-36 score* } \\
\hline Physical function & $35(5-100)$ & $40(15-80)$ & $35(5-100)$ & $45(5-85)$ & $67.5(20-95)$ & $55(5-95)$ & $<0.001$ & 0.033 & $<0.001$ \\
\hline Social function & $50(12.5-100)$ & $75(25-100)$ & $50(12.5-100)$ & $62.5(25-100)$ & $81.2(25-100)$ & $75(25-100)$ & $<0.001$ & 0.036 & $<0.001$ \\
\hline $\begin{array}{l}\text { Physical role } \\
\text { limitation }\end{array}$ & $0(0-100)$ & $0(0-100)$ & $0(0-100)$ & $100(0-100)$ & $100(0-100)$ & $100(0-100)$ & $<0.001$ & 0.068 & $<0.001$ \\
\hline $\begin{array}{l}\text { Emotional role } \\
\text { limitation }\end{array}$ & $0(0-100)$ & $100(0-100)$ & $100(0-100)$ & $100(0-100)$ & $100(0-100)$ & $100(0-100)$ & $<0.001$ & 0.348 & $<0.001$ \\
\hline Bodily pain & $35(0-70)$ & $47.5(12.5-67)$ & $35(0-100)$ & $56.2(10-100)$ & $57.5(32.5-90)$ & $57.5(15-100)$ & $<0.001$ & 0.010 & $<0.001$ \\
\hline Mental health & $64(32-88)$ & $68(32-72)$ & $64(32-88)$ & 72 (28-92) & $64(28-84)$ & 72 (28-92) & $<0.001$ & 1.000 & 0.001 \\
\hline Vitality & $35(10-75)$ & $50(30-70)$ & $40(10-75)$ & $50(15-80)$ & $55(5-65)$ & $50(5-50)$ & $<0.001$ & 0.571 & $<0.001$ \\
\hline $\begin{array}{l}\text { Health percep- } \\
\text { tion }\end{array}$ & $35(15-85)$ & $50(20-60)$ & $40(15-85)$ & $50(15-80)$ & $55(20-65)$ & $55(15-80)$ & $<0.001$ & 0.025 & $<0.001$ \\
\hline GDS category, n (\%) & & & & & & & $<0.001$ & $N / T$ & $<0.001$ \\
\hline No depression & $31(38.2)$ & $14(82.4)$ & $45(45.9)$ & $58(76.3)$ & 25(96.2) & $83(81.4)$ & & & \\
\hline $\begin{array}{l}\text { Possible depres- } \\
\text { sion }\end{array}$ & $12(14.9)$ & $1(5.8)$ & $13(13.3)$ & $12(15.8)$ & - & $12(11.8)$ & & & \\
\hline Depression & $38(46.9)$ & $2(11.8)$ & $40(40.8)$ & $6(7.9)$ & $1(3.8)$ & $7(6.8)$ & & & \\
\hline \multicolumn{10}{|l|}{$\begin{array}{l}\text { Knee pain VAS } \\
\text { score* }\end{array}$} \\
\hline At rest & $4(0-10)$ & $2(0-8)$ & $3.5(0-10)$ & $2(0-6)$ & $0(0-6)$ & $2(0-6)$ & $<0.001$ & 0.040 & $<0.001$ \\
\hline Walking & $8(2-10)$ & $7(4-10)$ & $8(2-10)$ & $7(4-10)$ & $6(3-10)$ & $6.5(3-10)$ & $<0.001$ & 0.009 & $<0.001$ \\
\hline
\end{tabular}

$P$-value less than $0.05(p<0.05)$ is statistically significant

$\mathrm{p}_{\mathrm{F}}$; significance of difference between females in Group 1 and Group 2. $\mathrm{p}_{\mathrm{M}}$; significance of difference between males in Group 1 and Group 2. $\mathrm{p}_{\mathrm{G}}$; significance of difference between Group 1 and Group 2

$n$ number

${ }^{*}$ Given as median (min-max)

neuropathic mechanisms, with additional features such as allodynia, increased sensitivity to sound and light, sleep disturbances, fatigue, and cognitive problems. It is now called nociplastic pain [28], which probably stands as a third component of the pain in osteoarthritis. Pain, which was initially responsive to analgesics and nonsteroidal anti-inflammatory drugs, may change character and gain long-lasting, more extensive and refractory features in the later course of the disease [6, 7]. At that stage, centrally acting agents, such as duloxetin and pregabalin, may work $[7,29,30]$. So characterization of the pain in osteoarthritis is of importance for proper management and patient care.

A wide range of frequency of neuropathic pain, 5.4$52 \%$, was reported in knee osteoarthritis [8-12]. The ranges of the age (44-81 to 67-99 years) and symptom 
Table 4 Fall risk, stability and Fourier harmony indices obtained by the computerized posturography in eight positions

\begin{tabular}{|c|c|c|c|c|c|c|c|c|c|}
\hline & \multicolumn{3}{|l|}{ Group 1} & \multicolumn{3}{|l|}{ Group 2} & \multirow[t]{2}{*}{$p_{F}$} & \multirow[t]{2}{*}{$\mathrm{p}_{\mathrm{M}}$} & \multirow[t]{2}{*}{$p_{G}$} \\
\hline & Female $(n=81)$ & Male $(n=17)$ & Total $(n=98)$ & Female $(n=76)$ & Male $(n=26)$ & Total $(n=102)$ & & & \\
\hline $\begin{array}{l}\text { Fall risk cat- } \\
\text { egory, n (\%) }\end{array}$ & & & & & & & 0.841 & 0.029 & 0.502 \\
\hline Low & $19(23.4)$ & $1(5.9)$ & $20(20.4)$ & $17(22.3)$ & $11(42.3)$ & $28(27.5)$ & & & \\
\hline Intermediate & $23(28.4)$ & $5(29.4)$ & $28(28.6)$ & $19(25)$ & $6(23)$ & $25(24.5)$ & & & \\
\hline High & $39(48.2)$ & $11(64.7)$ & $50(51)$ & $40(52.7)$ & $9(34.7)$ & $49(48)$ & & & \\
\hline \multicolumn{10}{|l|}{ Stabililty index* } \\
\hline Position 1 & $21.5(9.6-90.5)$ & $24.6(15.5-90.6)$ & 22 (9.6-90.6) & $18.6(8.9-114.2)$ & $19.4(11.3-70)$ & $19(8.9-114.2)$ & 0.202 & 0.164 & 0.132 \\
\hline Position 2 & $21.2(10.4-75.5)$ & $29(17.6-98.6)$ & $22.7(10.4-98.6)$ & $23(8.5-76.5)$ & $19.4(9-103.8)$ & $21.7(8.5-103.8)$ & 0.568 & $<0.001$ & 0.047 \\
\hline Position 3 & $16.2(9.6-43.1)$ & $19.4(10.7-53.8)$ & $16.5(9.6-53.8)$ & 15 (5.6-76.8) & $14.4(9.4-53.8)$ & $14.7(5.6-76.8)$ & 0.035 & 0.008 & 0.002 \\
\hline Position 4 & $22(8.7-69.1)$ & $25.1(15.7-75.1)$ & $22.4(8.7-75.1)$ & $19.6(6.9-59.2)$ & $19.6(10.6-56.9)$ & $19.6(6.9-59.2)$ & 0.270 & 0.021 & 0.031 \\
\hline Position 5 & $23.1(6.1-77)$ & $33.9(16.8-109.3)$ & $24.3(6.1-109.3)$ & $23.1(8.7-66.5)$ & $21.7(10.7-76.5)$ & $22.5(8.7-76.5)$ & 0.907 & 0.031 & 0.312 \\
\hline Position 6 & $23(5.5-90.3)$ & $27.7(19.6-73.3)$ & $23.4(5.5-90.3)$ & $25.5(9.6-60.3)$ & $20.5(11.8-78.9)$ & $23.8(9.6-78.9)$ & 0.521 & 0.003 & 0.441 \\
\hline Position 7 & $23.7(7.6-82.1)$ & $33(13.8-78.1)$ & $24.4(7.6-82.1)$ & $24.9(6.4-60.2)$ & 19.9 (12.8-91.9) & $23.9(6.4-91.9)$ & 0.769 & 0.007 & 0.287 \\
\hline Position 8 & $24.9(6.1-130.2)$ & $32(4.7-80.2)$ & $26.8(4.7-130.2)$ & $25.1(5.9-75.5)$ & $19.5(12.1-92.7)$ & $22.6(5.9-92.7)$ & 0.355 & 0.005 & 0.013 \\
\hline \multicolumn{10}{|c|}{$\begin{array}{l}\text { Fourier harmony } \\
\text { index* }\end{array}$} \\
\hline Position 1 & $0.91(0.25-1)$ & $0.77(0.29-0.99)$ & $0.88(0.25-1)$ & $0.89(0.33-1)$ & $0.79(0.39-0.99)$ & $0.87(0.33-1)$ & 0.781 & 0.559 & 0.791 \\
\hline Position 2 & $0.77(0.23-0.99)$ & $0.76(0.22-0.98)$ & $0.77(0.22-0.99)$ & $0.85(0.11-0.99)$ & $0.75(0.4-0.97)$ & $0.84(0.11-0.99)$ & 0.020 & 0.551 & 0.028 \\
\hline Position 3 & $0.87(0.22-1)$ & $0.85(0.34-0.97)$ & $0.86(0.22-1)$ & $0.88(0.32-0.99)$ & $0.90(0.6-0.99)$ & $0.89(0.32-0.99)$ & 0.239 & 0.123 & 0.072 \\
\hline Position 4 & $0.85(0.17-0.99)$ & $0.72(0.31-0.97)$ & $0.84(0.17-1)$ & $0.86(0.26-0.99)$ & $0.84(0.28-0.99)$ & $0.85(0.26-0.99)$ & 0.768 & 0.146 & 0.839 \\
\hline Position 5 & $0.90(0.42-0.99)$ & $0.90(0.28-0.98)$ & $0.90(0.28-0.99)$ & $0.89(0.22-0.99)$ & $0.87(0.45-0.98)$ & $0.88(0.22-0.99)$ & 0.499 & 0.950 & 0.436 \\
\hline Position 6 & $0.88(0.38-0.99)$ & $0.88(0.51-0.98)$ & $0.88(0.38-0.99)$ & $0.88(0.51-0.98)$ & $0.93(0.48-0.99)$ & $0.89(0.28-0.99)$ & 0.545 & 0.164 & 0.172 \\
\hline Position 7 & $0.89(0.23-0.99)$ & $0.94(0.48-0.98)$ & $0.90(0.23-0.99)$ & $0.88(0.28-0.99)$ & $0.88(0.52-0.99)$ & $0.88(0.5-0.99)$ & 0.519 & 0.636 & 0.382 \\
\hline Position 8 & $0.88(0.03-0.99)$ & $0.86(0.49-0.98)$ & $0.88(0.03-0.99)$ & $0.86(0.16-0.98)$ & $0.85(0.45-0.99)$ & $0.86(0.16-0.99)$ & 0.570 & 0.891 & 0.696 \\
\hline
\end{tabular}

$P$-value less than $0.05(p<0.05)$ is statistically significant

$\mathrm{p}_{\mathrm{F}}$; significance of difference between females in Group 1 and Group 2. $\mathrm{p}_{\mathrm{M}}$; significance of difference between males in Group 1 and Group 2. $\mathrm{p}_{\mathrm{G}}$; significance of difference between Group 1 and Group 2. Positions: 1, stable surface/eyes open/face forward; 2, stable surface/eyes closed/face forward; 3 , unstable surface/eyes open/face forward; 4 , unstable surface/eyes closed/face forward; 5 , stable surface/eyes closed/head rotated $45^{\circ}$ to the right; 6 , stable surface/eyes closed/head rotated $45^{\circ}$ to the left; 7 , stable surface/eyes closed/head tilted back $30^{\circ}$; and 8 , stable surface/eyes closed/head tilted $30^{\circ}$ forward

$n$ number

* Given as median (min-max)

duration (less than 1 year to 8.3 years) were quite different in these studies. Moreover, the assessment tools for neuropathic pain were different, as well. There is no gold standard tool for neuropathic pain. Three commonly used tools are painDETECT, LANSS, and DN-4 $[12,26]$. The first is based on the self-reported symptoms and originally designed for patients with low back pain although modified for knee osteoarthritis [12]. DN-4 is shorter, easier to perform, and was previously reported to be more reliable than LANSS in terms of sensitivity and specificity in Turkish population [26]. Although these tools give different prevalences of neuropathic pain in knee osteoathritis, the estimate of neuropathic pain was found to be unchanged by the questionnaire type in a meta-analysis [31]. But the validity of neuropathic pain questionnaires, selection bias, methodological quality and study heterogeneity were of great concern and standardized criteria for neuropathic pain were suggested to be developed [31].

Although higher age and longer symptom duration were found to be associated with neuropathic pain in this study, these were not uniform among previous studies [8-13]. Conflicting results were possibly due to heterogeneity of the study populations. We identified a relationship between neuropathic pain and lower education in knee osteoarthritis (Table 1). Education status was not found to be associated with neuropathic pain in two previous studies, one of which was conducted in Turkish population $[11,12]$. It may be an important point that both studies assessed neuropathic pain with painDETECT, a self-reported questionnaire.

As demonstrated in Table 2, and previously reported [10], knee osteoarthritis patients with neuropathic pain were slightly shorter in stature and had slightly higher body mass index and body fat percentage. These slight 
but significant differences are prone to type II error to be detected in smaller-scale studies [11]. Higher body mass index may be speculated to be associated with higher osteoarthritis severity, which in turn, may contribute to neuropathic pain (Table 3). Observations of beneficial effect of even slight reductions in body mass index and fat on osteoarthritis severity and symptoms may support this speculation [32].

Unfavorable osteoarthritis severity index, radiological class and postural deviation in the knee osteoarthritis patients with neuropathic pain may reflect an underlying pathophysiological relationship between osteoarthritis severity and neuropathic pain particularly if considered together with longer symtom duration and higher age in patients with neuropathic pain (Tables 1 and 3). Worse physical function tests and quality of life in those patients with neuropathic pain is possibly the result of that association but potentially has intrinsic impact on the mood, pain perception, and cognition, which may be related to central sensitization [5-7], as well. Similar results were previously reported $[5,11]$ along with conflicting ones $[9,11,13,33]$, again probably due to the differences in assessment tools and methodology, patient heterogeneity, and the lack of standardization.

Although fall risk was similar in knee osteoarthritis patients with or without neuropathic pain, stability and Fourier harmony indices were worse in the neuropathic pain group especially when the visual and/or proprioceptive input was blocked (Table 4). This was a novel finding and may be related to more severe osteoarthritis in the neuropathic pain group.

Increased pain perception, presence of painful areas other than the knee, and more prevalent depression in knee osteoarthritis patients with neuropathic pain indicate a more generalized psychopathological state rather than isolated neuropathic pain and may explain vulnerability to secondary hyperalgesia (Tables 1 and 3). Similar to our results, more prevalent comorbid diseases, higher number of drugs used, and a worse metabolic profile were previously reported in knee osteoarthritis patients with neuropathic pain [10]. Importantly, the sex was a significant influencer of the study results, although the frequency of female sex was similar in knee osteoarthritis patients with or without neuropathic pain.

Primary limitation of this study is the cross-sectional design that holds us to provide causal relationships regarding the study results. Type II error is also of concern in between-group male comparisons.

\section{Conclusions}

In conclusion, almost half of the patients with knee osteoarthritis had neuropathic pain which was associated with longer symptom duration and higher age, lower education, higher body mass index, more severe radilogical findings, worse pain perception, lower physical function and quality of life, and lower stability. Sex has an apparent effect on the study results. The lack of a gold standard assessment tool for neuropathic pain and a standardized methodology in knee osteoarthritis complicates comparison of the results of different studies.

\section{Abbreviations \\ WOMAC: Western Ontario and McMaster Osteoarthritis Index; SF-36: Short form-36; DN-4: Douleur neuropathique-4; VAS: Visual analogue scale; LANSS: Leeds assessment of neuropathic symptoms and signs pain scale. \\ Acknowledgements \\ None. \\ Authors' contributions \\ Concept and design: UGD, AND, NFT. Data collection and processing: UGD, analysis and interpretation: UGD, literature review: UGD, NFT, writing: UGD, AND. Critical review: NFT. All authors read and approved the final manuscript. \\ Funding \\ This study did not receive support from any funding source. \\ Availability of data and materials \\ The derived data generated in this research will be shared on reasonable request to the corresponding author.}

\section{Declarations}

Ethics approval and consent to participate

Written informed consent was obtained from each patient. Institutional Review Board of Antalya Training and Research Hospital approved the study (Number: 1/2-11.01.2018). All procedures performed in studies involving human participants were in accordance with the ethical standards of the institutional and/or national research committee and with the 1964 Helsinki declaration and its later amendments or comparable ethical standards.

Consent for publication

Not applicable.

\section{Competing interests}

The authors declare that they have no competing interests.

\section{Author details}

'Department of Physical Therapy and Rehabilitation, University of Health Sciences, Antalya Training and Research Hospital, 07100 Muratpaşa, Antalya, Turkey. ${ }^{2}$ Department of Physical Medicine and Rehabilitation Rheumatology Clinic, Faculty of Medicine, Akdeniz University, 07100 Antalya, Turkey. ${ }^{3}$ Department of Physical Therapy and Rehabilitation, University of Health Sciences, Antalya Training and Research Hospital, 07100 Antalya, Turkey.

Received: 20 August 2021 Accepted: 25 October 2021

Published online: 07 November 2021

References

1. Pereira D, Peleteiro B, Araujo J, Branco J, Santos RA, Ramos E, et al. The effect of osteoarthritis definition on prevalence and incidence estimates: a systematic review. Osteoarthr Cartil. 2011;19:1270-85. https://doi.org/ 10.1016/j.joca.2011.08.009.

2. Corti MC, Rigon C. Epidemiology of osteoarthritis: prevalence, risk factors, and functional impact. Aging Clin Exp Res. 2003;15:359-63. https://doi. org/10.1007/BF03327356. 
3. Kaçar C, Gilgil E, Urhan S, Arıkan V, Dündar Ü, Oksuz MC, et al. The prevalence of symptomatic knee and distal interphalangeal joint osteoarthritis in the urban population of Antalya, Turkey. Rheumatol Int. 2005;25:201-4. https://doi.org/10.1007/s00296-003-0415-z.

4. Ivanavicius SP, Ball AD, Heapy CG, Westwood FR, Murray F, Read SJ. Structural pathology in a rodent model of osteoarthritis is associated with neuropathic pain: increased expression of ATF-3 and pharmacological characterisation. Pain. 2007;128:272-82. https://doi.org/10.1016/j.pain. 2006.12.022.

5. Hochman JR, Davis AM, Elkayam J, Gagliese L, Hawker GA. Neuropathic pain symptoms on the modified painDETECT correlate with signs of central sensitization in knee osteoarthritis. Osteoarthr Cartil. 2013;21:123642. https://doi.org/10.1016/j.joca.2013.06.023.

6. Schaible HG. Mechanisms of chronic pain in osteoarthritis. Curr Rheumatol Rep. 2012;14:549-56. https://doi.org/10.1007/s11926-012-0279-x.

7. Dimitroulas T, Duarte RV, Behura A, Kitas GD, Raphael JH. Neuropathic pain in osteoarthritis: a review of pathophysiological mechanisms and implications for treatment. Semin Arthritis Rheum. 2014:44:145-54. https://doi.org/10.1016/j.semarthrit.2014.05.011.

8. Oteo-Álvaro Á, Ruiz-Ibán MA, Miquens X, Stern A, Villoria J, SánchezMagro I, et al. High prevalence of neuropathic pain features in patients with knee osteoarthritis: a cross-sectional study. Pain Pract. 2015;15:61826. https://doi.org/10.1111/papr.12220.

9. Ohtori S, Orita S, Yamashita M, Ishikawa T, Ito T, Shigemura T, et al. Existence of a neuropathic pain component in patients with osteoarthritis of the knee. Yonsei Med J. 2012;53:801-5. https://doi.org/10.3349/ymj.2012. 53.4.801.

10. Fernandes GS, Valdes AM, Walsh DA, Zhang W, Doherty M. Neuropathiclike knee pain and associated risk factors: a cross-sectional study in a UK community sample. Arthritis Res Ther. 2018;27:215-21. https://doi.org/10. 1186/s13075-018-1717-6.

11. Polat CS, Dogan A, Sezgin Ozcan D, Koseoglu BF, Kocer Akselim S. Is there a possible neuropathic pain component in knee osteoarthritis? Arch Rheumatol. 2017;32:333-8. https://doi.org/10.5606/ArchRheumatol.2017. 6006.

12. Hochman JR, Gagliese L, Davis AM, Hawker GA. Neuropathic pain symptoms in a community knee OA cohort. Osteoarthr Cartil. 2011;19:647-54. https://doi.org/10.1016/j.joca.2011.03.007.

13. Hochman JR, French MR, Berminghamet SL, Hawker GA. The nerve of osteoarthritis pain. Arthrit Care Res. 2010;62:1019-23. https://doi.org/10. 1002/acr.20142

14. Bellamy N, Buchanan WW, Goldsmith CH, Campbell J, Stitt LW. Validation study of WOMAC: a health status instrument for measuring clinically important patient relevant outcomes to antirheumatic drug therapy in patients with osteoarthritis of the hip or knee. J Rheumatol. 1988;15:1833-40.

15. Tüzün E, Eker L, Aytar A, Daşkapan A, Bayramoğlu M. Acceptability, reliability, validity and responsiveness of the Turkish version of WOMAC osteoarthritis index. Osteoarthr Cartil. 2005;13:28-33. https://doi.org/10. 1016/j.joca.2004.10.010

16. Ware JE, Sherbourne CD. The Mos 36 item short-form health survey (SF-36). I. Conceptual framework and item selection. Med Care. 1992;30:473-83.

17. Koçyiğit H, Aydemir Ö, Fişek G, Ölmez N, Memiş A. Kısa Form-36 (KF36)'nın Türkçe Versiyonunun güvenilirliği ve geçerliliği. İlaç ve Tedavi Dergisi. 1999;12:102-6 ((Turkish)).

18. Yesavage JA, Brink TL, Rose TL, Lum O, Huang V, Adey M, et al. Development and validation of a geriatric depression screening scale: a preliminary report. Psychiatr Res. 1982;17:37-49. https://doi.org/10.1016/ 0022-3956(82)90033-4.

19. Ertan T, Eker E, Şar V. Geriatrik depresyon ölçeğinin Türk yaşıı nüfusunda geçerlilik ve güvenilirliği. Nöropsikiyatri Arşivi. 1997;34:62-71 ((Turkish)).

20. Jones CJ, Rikli RE, Beam WC. A 30-s chair-stand test as a measure of lower body strength in community-residing older adults. Res Q Exerc Sport. 1999;70:113-9. https://doi.org/10.1080/02701367.1999.10608028.

21. Bolton JWR, Weiman DS, Haynes J, Hornung CA, Olsen GN, Almond CH. Stair climbing as an indicator of pulmonary function. Chest. 1987:92:7838. https://doi.org/10.1378/chest.92.5.783.

22. [No authors listed]. ATS statement: guidelines for the six-minute walk test. Am J Respir Crit Care Med. 2002;166:111-7. https://doi.org/10.1164/ ajrccm.166.1.at1102.

23. Kohen-Raz R. Application of tetra-ataxiametric posturography in clinical and developmental diagnosis. Percept Mot Skill. 1991;73:635-56. https:// doi.org/10.2466/pms.1991.73.2.635.

24. Friedrich M, Grein HJ, Wicher C, Schuetze J, Mueller A, Lauenroth A, et al. Influence of pathologic and simulated visual dysfunctions on the postural system. Exp Brain Res. 2008;186:305-14. https://doi.org/10.1007/ s00221-007-1233-4

25. Bouhassira D, Attal N, Alchaar H, Boureau F, Brochet B, Bruxelle J, et al. Comparison of pain syndromes associated with nervous or somatic lesions and development of a new neuropathic pain diagnostic questionnaire (DN-4). J Pain. 2005;114:29-36. https://doi.org/10.1016/j.pain. 2004.12.010.

26. Unal-Cevik I, Sarioglu-Ay S, Evcik D. A comparison of the DN-4 and LANSS questionnaires in the assessment of neuropathic pain: validity and reliability of the Turkish version of DN-4. J Pain. 2010;11:1129-35. https://doi. org/10.1016/j.jpain.2010.02.003.

27. Kellgren JH, Lawrence JS. Radiological assessment of osteo-arthrosis. Ann Rheum Dis. 2000;16:494-502. https://doi.org/10.1136/ard.16.4.494.

28. Kosek E, Clauw D, Nijs J, Baron R, Gilron I, Harris RE, et al. Chronic nociplastic pain affecting the musculoskeletal system: clinical criteria and grading system. Pain. 2021;162:2629-34. https://doi.org/10.1097/j.pain.00000 00000002324.

29. Chappell AS, Desaiah D, Seifert HL, Zhang S, Skljarevski V, Belenkov Y, et al. A double-blind, randomized, placebo-controlled study of the efficacy and safety of duloxetine for the treatment of chronic pain due to osteoarthritis of the knee. Pain Pract. 2011;11:33-41. https://doi.org/10.1111/j. 1533-2500.2010.00401.x

30. Sofat N, Harrison A, Russell MD, Ayis S, Kiely PD, Baker EH, et al. The effect of pregabalin or duloxetine on arthritis pain: a clinical and mechanistic study in people with hand osteoarthritis. J Pain Res. 2017;10:2437-49. https://doi.org/10.2147/jpr.s147640.

31. French HP, Smart KM, Doyle F. Prevalence of neuropathic pain in knee or hip osteoarthritis: a systematic review and meta-analysis. Semin Arthritis Rheum. 2017;47:1-8. https://doi.org/10.1016/j.semarthrit.2017.02.008.

32. Bliddal H, Leeds AR, Christensen R. Osteoarthritis, obesity and weight loss: evidence, hypotheses and horizons-a scoping review. Obes Rev. 2014;15:578-86. https://doi.org/10.1111/obr.12173.

33. Narayan RV, Thabah MM, Poduval M. Neuropathic pain among patients with primary knee osteoarthritis: results of a cross sectional study from a tertiary care centre in Southern India. Indian J Rheumatol. 2017;12:132-8. https://doi.org/10.4103/injr.injr_90_16.

\section{Publisher's Note}

Springer Nature remains neutral with regard to jurisdictional claims in published maps and institutional affiliations. 